\title{
Expectativas e sentimentos de familiares cuidadores de crianças com necessidades especiais que realizam equoterapia
}

\author{
Expectations and feelings of family caregivers of children with special needs \\ performing equotherapy
}

\begin{abstract}
Luiza Tarsila do Vale Pereira ${ }^{1}$, Charlene de Oliveira Pereira², Paula Christianne Gomes Gouveia Souto Maia $^{3}$, Milena Nunes Alves de Sousa ${ }^{4 *}$

${ }^{1}$ Acadêmica de Medicina do Centro Universitário de Patos - UNIFIP - Patos-PB; ${ }^{2}$ Psicóloga. Mestranda em Ciências da Saúde pela Faculdade de Medicina do ABC. Docente no Curso de Medicina do Centro Universitário de Patos UNIFIP - Patos-PB; ${ }^{3}$ Médica. Doutoranda em Ciências da Saúde pela Faculdade de Medicina do ABC. Docente e Coordenadora do Curso de Medicina do Centro Universitário de Patos - UNIFIP - Patos-PB; ${ }^{4}$ Doutora e Pós-Doutora em Promoção de Saúde. Docente no Curso de Medicina do Centro Universitário de Patos - UNIFIP - Patos-PB.
\end{abstract}

\begin{abstract}
Resumo
Objetivo: avaliar as expectativas e sentimentos de familiares cuidadores de crianças com necessidades especiais que realizam equoterapia. Metodologia: pesquisa de campo, com caráter descritivo e com abordagem quantitativa. $\mathrm{O}$ estudo aconteceu na cidade de Patos, localizada no sertão da Paraíba, com 18 familiares cuidadores $(94,74 \%)$ de crianças assíduas às atividades do Centro de Equoterapia. $\mathrm{O}$ instrumento utilizado para a coleta de dados validado em português foi o "Questionário para a Família" e duas questões adicionais elaboradas pela pesquisadora. Os dados foram analisados a partir da estatística descritiva. Resultados: o convívio com crianças portadoras de necesidade especiais gera expectativas quanto ao bem-estar dos filhos $(88,9 \%)$, sucesso na escola $(50,0 \%)$, autonomia na vida diária $(72,2 \%)$, felicidade $(66,7 \%)$, normalidade $(66,7 \%)$, cuidados pessoais $(50,0 \%)$. Quanto aos sentimentos, destacou-se a ansiedade $(44,4 \%)$, sentido de proteção $(55,6 \%)$ e tristeza (67\%). Conclusão: cuidar de crianças portadoras de necessidades especiais gera nos cuidadores uma série de sentimentos e expectativas, sendo estes causados por dilemas e dúvidas em relação à vida e ao futuro da criança.

Palavras-chave: Pessoas com Deficiência. Crianças. Terapias Complementares.
\end{abstract}

\begin{abstract}
Objective: to evaluate the expectations and feelings of family caregivers of children with special needs who undergo horse therapy. Methodology: descriptive field research, with quantitative approach. The study took place in the city of Patos, located in the countryside of Paraiba, with 18 family members (94.74\%) that took care of children regularly attending the activities of the Equine Therapy Center. The instrument used for data collection was the "Family Questionnaire", validated in Portuguese, with two additional questions elaborated by the researcher. Data were analysed from the descriptive statistics. Results: living with children with special need raises expectations about the welfare of children (88.9\%), success in school (50.0\%), autonomy in daily life (72.2\%), happiness (66.7\%), normal (66.7\%), personal care (50.0\%); As for the feelings, anxiety (44.4\%), sense of protection (55.6\%) and sadness (67\%). Conclusion: caring for children with special needs is associated with a series of feelings and expectations caused by dilemmas and doubts regarding the life and future of their children.

Keywords: Disabled Persons. Child. Complementary Therapies.
\end{abstract}

\section{INTRODUÇÃO}

A assistência neonatal avançou muito nas últimas décadas. Transformações econômicas, políticas, sociais e culturais produzidas pela sociedade, com a introdução de recursos terapêuticos e tecnológicos mais eficazes e recursos humanos especializados. Este progresso colabora com o aumento dos índices de crianças com doenças crônicas/incapacitantes, dentre as quais muitas requerem atenção especial para manter sua sobrevivência (MANOEL

Correspondente/ Corresponding: *Milena Nunes Alves de Sousa - End: Rua Horácio Nóbrega, SN, Belo Horizonte, Patos - PB, CEP: 58704-000.

-Tel.: (83) 3421-7300. - Email: minualsa@hotmail.com. et al., 2013; NEVES; SILVEIRA, 2013).

Dentre tais agravos, tem-se aquele conjunto que possuem riscos maiores por apresentarem condições físicas, comportamentais, desenvolvimentais ou emocionais crônicas, chamado de indivíduos com necessidades especiais de saúde (FIGUEIREDO; SOUSA; GOMES, 2016).

De acordo com o CID-10, deficiência define-se por uma disfunção no desempenho psicológico, anatômico ou fisiológico, podendo relacionar-se com um desequilíbrio no estado do órgão. Tratando-se de incapacidades seria, portanto, o resultado da deficiência que implica inabilita o indivíduo para desempenhar uma tarefa que normalmente pode ser desenvolvida por outra pessoa ausente desse 
déficit. A deficiência em conjunto com a incapacidade de desenvolver algo, acaba gerando desvantagens, pois esse grupo é, muitas vezes, limitado a desenvolver um papel que é natural para o ser humano. Tais desvantagens irão refletir em sua performance em relação aos demais grupo sociais (SILVA; PANHOCA; BLACHMAN, 2004).

Considerando as especificidades daqueles que são designados como indivíduos com necessidades especiais, o tratamento não propicia cura, mas atua na melhora do prognóstico inerente a limitação e também é coadjuvante sobre os níveis de qualidade de vida. Dentre as alternativas terapêuticas, a equoterapia tem se destacado como um método alternativo extremamente benéfico. $A$ técnica utiliza o cavalo como motivador para o tratamento, por se tratar de um animal dócil, de porte e força, que permite o manuseio e montaria (ANDRADE; CUNHA, 2014; BARBOSA; VAN MUNSTER, 2013; TOIGOA; JÕNIORB; ÁVILA, 2008).

Portanto, contribui com ganhos físicos, psicológicos e educacionais, já que precisa da participação do corpo inteiro, possibilitando o desenvolvimento da força, tônus muscular, flexibilidade, relaxamento, conscientização do próprio corpo e aperfeiçoamento da coordenação motora e do equilíbrio (ANDRADE; CUNHA, 2014).

A criança com necessidades especiais que pratica de equoterapia recebe grande quantidade de estímulos sensitivos que atingem os sistemas de propriocepção, tátil, vestibular e visual, entre outros. As sessões de equitação além de eficazes são motivadoras e recreativas (BARBOSA; VAN MUNSTER, 2013; CHOON, 2015).

Apesar de alternativas viáveis, a exemplo da equoterapia, as quais permeiam bons resultados junto a estas crianças, o fato é que a família e/ou cuidadores vivenciam sentimentos e realidades muitas vezes contraditórias. 0 que os familiares cuidadores experienciam no "início da dependência [...] evidenciam o processo de adaptação da família à situação [...] [e] se manifesta de múltiplas formas" (MARTINS; MONTEIRO; GONÇALVES, 2016, p. 1117).

Dentro deste contexto, objetivou-se avaliar as expectativas e sentimentos de familiares cuidadores de crianças com necessidades especiais que realizam equoterapia, uma vez que se julga como de grande importância científica e social, já que é necessário incentivar políticas públicas acessíveis para prover o tratamento multiprofissional para estes infantes e promover o bem-estar do grupo, bem como dar suporte aos familiares cuidadores, que muitas vezes estão devastados fisica e psicologicamente. Afinal, passam "por diferentes fases, das quais decorrem diferentes necessidades de suporte com vistas a atingir o equilíbrio" (MARTINS; MONTEIRO; GONÇALVES, 2016, p. 1117).

\section{METODOLOGIA}

Pesquisa de campo, de caráter descritivo e com abordagem quantitativa. $O$ estudo aconteceu no município de Patos, no sertão da Paraíba, no Centro de Equoterapia
(EQUOPATOS). Este cenário foi idealizado por Rivânio Sousa no ano de 2015 , que a partir da sua paixão por cavalos, estudou o tratamento e os benefícios do mesmo para a vida de pessoas com necessidades especiais. A idade das crianças atendidas varia entre 2 e 16 anos e as necessidades especiais contemplam: paralisia cerebral, transtornos do espectro autista e síndrome de Down. A maioria dos cuidadores consiste nos pais das crianças que acompanham as sessões de equoterapia e são voluntários no local.

Assim sendo, o universo de pesquisa era composto de 19 cuidadores que frequentavam regularmente (três vezes semanais) o centro citado, porém para a coleta de dados efetivou-se junto a 18 participantes, já que um cuidador não fora contactado para responder ao instrumento de coleta de dados, por não estar participando ativamente das sessões de equoterapia com a criança. Destaca-se que o lócus de pesquisa é o único da região que presta esse tipo de atendimento, por esta razão, não foi possível ampliar a amostra.

Os critérios de inclusão e exclusão utilizados para definir a amostra foram familiares cuidadores de crianças com necessidades especiais, com idade entre 02 a 16 anos, assíduos às atividades de equoterapia do Centro de Equoterapia em Patos - PB.

$\mathrm{O}$ instrumento utilizado para coleta de dados foi um questionário validado em português da Federação Nacional de Cooperativa de Solidariedade Social (FENACERCI, 2006), o qual se intitula de "Questionário para a Família" composto por 53 questionamentos. A escolha do mesmo deveu-se ao fato de possibilitar avaliar as expectativas e sentimentos de cuidadores em relação à vida das crianças com necessidades especiais que fazem algum tipo de terapia complementar, a equoterapia, por exemplo. Os questionamentos constam de dados pessoais do familiar cuidador e da criança, informações sobre o diagnóstico e os sentimentos dos pais no momento deste. Soma-se que o instrumento também contempla as expectativas dos pais quanto ao futuro da criança, entre outras. As questões apresentam respostas objetivas e variam em uma escala do tipo Linkert. Ainda, os pesquisadores adicionaram outros dois questionamentos, para verificar se a equoterapia propiciou melhorais/benefícios sobre a qualidade de vida da criança e o tipo de necessidade especial do infante.

Quanto à coleta de dados, a mesma foi realizada após a aprovação do projeto pelo Comitê de Ética em Pesquisa das Faculdades Integradas de Patos, conforme CAAE 79819917.0.0000.5181 e protocolo 2.404.740/2017.

Os resultados foram digitados em planilha do $\mathrm{Mi}$ crosoft Excel e o programa utilizado para a obtenção dos cálculos estatísticos foi o Statistical Package for the Social Sciences (SPSS) v. 23. Os dados foram analisados pelo método de análise descritiva quantitativa para idade dos pais e descritiva qualitativa de frequência para as demais variáveis. 


\section{RESULTADOS}

Participaram da pesquisa dezoito familiares cuidadores, destes $77,8 \%$ eram mães e $22,2 \%$ pais de crianças portadoras de necessidades especiais que frequentaram a EQUOPATOS. Quanto à idade do pai e da mãe, destaca-se que a média da idade paterna foi 4,68 anos mais elevada do que a materna, correspondendo a 36,72 anos e 32,06 anos, respectivamente.

A maioria das crianças $(83,3 \%$ ) possuía multideficiência e os $16,7 \%$ restantes foram diagnosticados com deficiência de linguagem. Em relação à necessidade especial, as respostas mais frequentes foram: "Transtorno do espectro autista" (55,6\%) e "Paralisia cerebral" (33,3\%) (Tabela 1).

Tabela 1 - Avaliação da deficiência e necessidade especial da criança praticamente de equoterapia

\begin{tabular}{lrc}
\hline Variáveis & $\mathbf{n}$ & $\%$ \\
\hline Deficiência do filho & & \\
\hline Linguagem & 3 & 16,7 \\
Multideficiência & 15 & 83,3 \\
Necessidade especial da criança & & \\
Paralisia cerebral & 6 & 33,3 \\
Transtorno do espectro autista & 10 & 55,6 \\
Tetralogia de fallot & 1 & 5,6 \\
\hline Meningomielocele & 1 & 5,6 \\
\hline
\end{tabular}

Fonte: Dados da pesquisa, 2019.

De acordo com a avaliação das emoções sentidas (sentimentos) relacionadas ao futuro do filho (Tabela 2), na avaliação da ansiedade o maior percentual correspondeu a frequentemente $(44,4 \%)$ na avaliação por si; e nunca ou algumas vezes na avaliação do parceiro com $27,8 \%$, cada; a maioria no sentido da proteção correspondeu à categoria constantemente, sendo 55,6\% por si e $61,1 \%$ pelo parceiro; para tristeza algumas vezes foi a mais ponderada, com $61,1 \%$ por si e $66,7 \%$ pelo parceiro; na avaliação da infelicidade as duas respostas mais enfatizadas foram: algumas vezes $(55,6 \%)$ por si e frequentemente $(50,0 \%)$ pelo parceiro.

$\mathrm{Na}$ opressão as duas maiores frequências foram na categoria nunca $(44,4 \%$ por si e $61,1 \%$ parceiro); na questão sem esperança a maioria foi na categoria nunca com 55,6\% em cada avaliação; na avaliação da insatisfação foi $55,6 \%$ na categoria algumas vezes em por si e foi $50,0 \%$ frequentemente na avaliação do parceiro; no item insegurança a maioria ocorreu na categoria frequentemente $(61,1 \%)$, por si e na avaliação do parceiro, as duas maiores frequências corresponderam as categorias frequentemente e nunca, cada uma com $38,9 \%$ (Tabela 2).
Tabela 2 - Avaliação das emoções dos pais/cuidadores em relação ao futuro do filho com necessidade especial praticamente de equoterapia

\begin{tabular}{|c|c|c|c|c|}
\hline \multirow{3}{*}{ Variável } & \multicolumn{4}{|c|}{ Pais } \\
\hline & \multicolumn{2}{|c|}{ Por si } & \multicolumn{2}{|c|}{ Parceiro } \\
\hline & $\mathbf{N}$ & $\%$ & $\mathrm{n}$ & $\%$ \\
\hline \multicolumn{5}{|l|}{ Ansiedade } \\
\hline Nunca & 1 & 5,6 & 5 & 27,8 \\
\hline Algumas vezes & 5 & 27,8 & 5 & 27,8 \\
\hline Frequentemente & 8 & 44,4 & 4 & 22,2 \\
\hline Constantemente & 4 & 22,2 & 4 & 22,2 \\
\hline \multicolumn{5}{|l|}{ Sentido de proteção } \\
\hline Nunca & - & - & & \\
\hline Algumas vezes & 2 & 11,1 & 1 & 5,6 \\
\hline Frequentemente & 6 & 33,3 & 6 & 33,3 \\
\hline Constantemente & 10 & 55,6 & 11 & 61,1 \\
\hline \multicolumn{5}{|l|}{ Tristeza } \\
\hline Nunca & 3 & 16,7 & 5 & 27,8 \\
\hline Algumas vezes & 11 & 61,1 & 12 & 66,7 \\
\hline Frequentemente & 2 & 11,1 & - & - \\
\hline Constantemente & 2 & 11,1 & 1 & 5,6 \\
\hline \multicolumn{5}{|l|}{ Infelicidade } \\
\hline Nunca & 1 & 5,6 & 2 & 11,1 \\
\hline Algumas vezes & 10 & 55,6 & 9 & 50,0 \\
\hline Frequentemente & 7 & 38,9 & 7 & 38,9 \\
\hline Constantemente & - & - & - & - \\
\hline \multicolumn{5}{|l|}{ Opressão } \\
\hline Nunca & 8 & 44,4 & 11 & 61,1 \\
\hline Algumas vezes & 5 & 27,8 & 6 & 33,3 \\
\hline Frequentemente & 4 & 22,2 & 1 & 5,6 \\
\hline Constantemente & 1 & 5,6 & - & - \\
\hline \multicolumn{5}{|l|}{ Sem esperança } \\
\hline Nunca & 10 & 55,6 & 10 & 55,6 \\
\hline Algumas vezes & 5 & 27,8 & 4 & 22,2 \\
\hline Frequentemente & 3 & 16,7 & 3 & 16,7 \\
\hline Constantemente & - & - & 1 & 5,6 \\
\hline \multicolumn{5}{|l|}{ Insatisfação } \\
\hline Nunca & 1 & 5,6 & 3 & 16,7 \\
\hline Algumas vezes & 10 & 55,6 & 5 & 27,8 \\
\hline Frequentemente & 7 & 38,9 & 9 & 50,0 \\
\hline Constantemente & - & - & 1 & 5,6 \\
\hline \multicolumn{5}{|l|}{ Insegurança } \\
\hline Nunca & 1 & 5,6 & 7 & 38,9 \\
\hline Algumas vezes & 4 & 22,2 & 3 & 16,7 \\
\hline Frequentemente & 11 & 61,1 & 7 & 38,9 \\
\hline Constantemente & 2 & 11,1 & 1 & 5,6 \\
\hline
\end{tabular}

Fonte: Dados da pesquisa, 2019.

Quanto a avaliação dos itens que julgam mais importantes para o futuro do filho (expectativas) (Gráfico 1), os percentuais variaram de $50,0 \%$ a $88,9 \%$ na categoria muito, em: reabilitação $(55,6 \%)$, bem-estar $(88,9 \%)$, sucesso na escola $(50,0 \%)$, autonomia na vida diária $(72,2 \%)$, felicidade $(66,7 \%)$, normalidade $(66,7 \%)$ e cuidados pessoais (50,0\%). No item experiência de vários tipos, o maior percentual $(44,4 \%)$ correspondeu à categoria muito; no 
item amigos em relações sociais as duas maiores frequências corresponderam a muito e bastante com percentuais de $38,9 \%$ e $33,3 \%$ respectivamente. Na satisfação e necessidade emocional, a maioria $(55,6 \%)$ considerou a categoria bastante; no item satisfação a necessidade sexual, 50,0\% indicou pouco; no item desporto as duas maiores frequências foram registradas na categoria um pouco $(38,9 \%)$ e muito $(27,8 \%)$.

Gráfico 1 - Avaliação dos itens que os pais/cuidadores julgam mais importantes para ofuturo do filho com necessidade especial praticamente de equoterapia

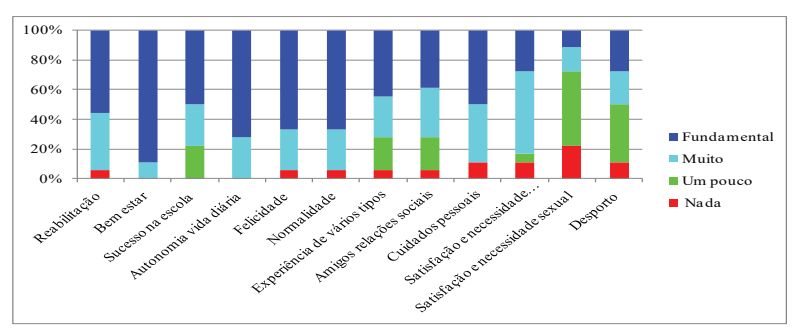

Fonte: Autoria própria

$\mathrm{Na}$ Tabela 3, quanto às expectativas relacionadas às redes de apoio mais relevantes para o futuro do filho, os dois maiores percentuais para si foram muito $(50,0 \%)$ e bastante $(38,9 \%)$ para a família; e para o parceiro a maioria foi bastante $(61,1 \%)$ também para a família. Para o item parceiro, o maior percentual foi muito tanto para si $(55,6 \%)$ ou para o seu parceiro $(50,0 \%)$. Ainda, a maioria respondeu muito em relação aos profissionais e ele próprio, com $55,6 \%$ para si ou para o seu parceiro; na avaliação dos amigos, os percentuais das categorias um pouco, bastante e muito, variaram de $27,8 \%$ a $38,9 \%$.

Tabela 3 - Expectativas relacionadas às redes de apoio que os pais/cuidadores consideram como mais relevantes ao futuro do filho com necessidade especial praticamente de equoterapia

\begin{tabular}{lrrrr}
\hline & \multicolumn{5}{c}{ Pais } \\
\cline { 2 - 6 } Variáveis & \multicolumn{2}{c}{ Para si } & \multicolumn{3}{c}{ Para o seu Parceiro } \\
\cline { 2 - 6 } & $\mathbf{N}$ & $\%$ & $\mathbf{n}$ & $\%$ \\
\hline Família & & & & \\
Nada & - & - & 1 & 5,6 \\
Um pouco & 2 & 11,1 & 5 & 27,8 \\
Bastante & 7 & 38,9 & 11 & 61,1 \\
Muito & 9 & 50,0 & 1 & 5,6 \\
O seu parceiro & & & & \\
Nada & 3 & 16,7 & 3 & 16,7 \\
Um pouco & 3 & 16,7 & 2 & 11,1 \\
Bastante & 2 & 11,1 & 4 & 22,2 \\
Muito & 10 & 55,6 & 9 & 50,0 \\
Profissionais & & & & \\
Nada & - & - & - & - \\
Um pouco & 3 & 16,7 & 2 & 11,1 \\
Bastante & 5 & 27,8 & 6 & 33,3 \\
Muito & 10 & 55,6 & 10 & 55,6 \\
Ele próprio & & & &
\end{tabular}

\begin{tabular}{lrrrr}
\hline & \multicolumn{5}{c}{ Pais } \\
\cline { 2 - 5 } Variáveis & \multicolumn{2}{c}{ Para si } & \multicolumn{2}{c}{ Para o seu Parceiro } \\
\cline { 2 - 5 } & \multicolumn{1}{c}{$\mathbf{N}$} & $\%$ & $\mathbf{n}$ & $\%$ \\
\hline Nada & - & - & - & - \\
Um pouco & 1 & 5,6 & 1 & 5,6 \\
Bastante & 7 & 38,9 & 7 & 38,9 \\
Muito & 10 & 55,6 & 10 & 55,6 \\
Amigos & & & & \\
Nada & - & - & 1 & 5,6 \\
Um pouco & 6 & 33,3 & 5 & 27,8 \\
Bastante & 7 & 38,9 & 7 & 38,9 \\
Muito & 5 & 27,8 & 5 & 27,8 \\
\hline
\end{tabular}

Fonte: Dados da pesquisa, 2019.

Quanto à indagação, "a equoterapia trouxe benefício para a qualidade de vida da criança?" A maioria $(77,8 \%)$ respondeu que sim, (Tabela 4).

Tabela 4-Avaliação de quanto à equoterapia trouxe de beneficio à qualidade de vida da criança com necessidades especiais

\begin{tabular}{lrr}
\hline Variáveis & $\mathbf{n}$ & \% \\
\hline Nenhuma & 1 & 5,6 \\
Pouca & 2 & 11,1 \\
Muita & 14 & 77,8 \\
Não respondeu & 1 & 5,6 \\
\hline
\end{tabular}

Fonte: Dados da pesquisa, 2019.

\section{DISCUSSÃO}

Em relação ao perfil dos familiares cuidadores, estudos nacionais (FIRMINO; SOUSA, 2013; GÓES; CABRAL, 2017; NOHARA et al., 2017; OLIVEIRA; MATSUKURA, 2013) e internacionais (DIGUISEPPI et al., 2016; SUSKIND et al., 2016) apontam que, na maioria dos casos, a mãe é a cuidadora principal, como visualizado nesta investigação, a qual é atribuída maior parte da demanda de cuidados fundamentais para a melhor qualidade de vida da criança com necessidade especial de saúde. Isso pode ser explicado, pelo fato do cuidar ser culturalmente um papel delegado à mulher (BARBOSA et al., 2016).

Por conseguinte, o grupo cujas características, capacidades e necessidades diferem dos demais, designa-se por indivíduos com necessidades especiais. Essas necessidades dizem respeito a um conjunto de fatores de risco ou de ordem intelectual, emocional e física, que podem afetar a capacidade de um indivíduo em atingir o seu potencial máximo (ZAMO; ZAMO, 2015).

Considerando a assertiva constatou-se, neste estudo, que a maioria das crianças possui multideficiência. Semelhante ao achado, outra pesquisa também enfocou as multideficiências (NIJS; VLASKAMP; MAES, 2016).

$O$ cuidado dispensado para crianças com multideficiência determina ações específicas, o que pode condicionar baixa expectativa por parte dos cuidadores familiares quanto ao futuro do filho. Afinal, as preocupações começam com questões básicas, tais como em qual escola ele 
vai estudar. Ao se falar em indivíduos com necessidades múltiplas, sabe-se que é um grupo que precisa de ajuda e recursos que, em geral, não estão disponíveis na escola, por exemplo. Portanto, entre aquelas crianças com maiores necessidades, as com multideficiências, o desafio a ser enfrentado já no contexto escolar é enorme. Este fato decorre das dificuldades múltiplas que as caracterizam (FIGUEIREDO; SOUSA; GOMES, 2016; MENDES, 2017).

As crianças e jovens com multideficiências encontram barreiras desde cedo por "não terem capacidade para, de forma autônoma, participar ativamente na construção de experiências nas suas vidas, pois apresentam [...] impedimentos de nível físico, sensorial, comunicativo, psicossocial, de saúde, que os remete para uma natural passividade aprendida" (MENDES, 2017, p. 129).

Por sua vez, o transtorno do espectro autista e paralisia cerebral foram às condições clínicas mais relatadas. Os achados corroboram com a pesquisa de Barbosa e Van Munster (2013) os quais evidenciaram que a maior parte das crianças que realizavam equoterapia possuía de paralisia cerebral ou transtornos intelectuais mais brandos.

As deficiências físicas, tais como paralisias, ausência de visão ou de membros, causam um efeito imediato mais intenso por serem mais visíveis. Já auditivas e mentais (síndrome de Down, transtorno do espectro autista, síndrome de West, entre outras) a depender do grau de acometimento são menos sentidas inicialmente. Essas necessidades especiais, consequentemente, causam déficits motores, comportamentais e de aprendizado, que restringem sua atuação no meio em que vivem e seu comportamento perante as atividades de vida diária tornando-as menos funcionais e dependentes de cuidados (PAVÃO, 2011).

O quadro de dependência que é gerado no momento em que uma criança tem o diagnóstico de alguma necessidade especial, gera na família expectativas, sentimentos e vivências de temor e desepero (FIRMINO; SOUSA, 2013). Também, o processo de cuidar de uma criança com necessidades especiais associado à alta carga de responsabilidade pode levar ao cansaço, isolamento, sobrecarga e consequente estresse dos cuidadores (OLIVEIRA; MATSUKURA, 2013).

A família passa a vivenciar uma desestruturação, em um primeiro momento, por estar acontecendo algo fora da rotina normal. Apesar disto, vê a necessidade de buscar novas alternativas para se reorganizar e reestruturar, especialmente, por que o cuidado destinado a esta criança com necessidade especial deve ser continuo e exige um cuidador em tempo integral (NEVES; SILVEIRA, 2013).

É neste cenário que buscar por alternativas complementares pode auxiliar para um melhor prognóstico. "A intervenção no âmbito das Necessidades [...] Especiais deve privilegiar atividades que se adequem às características, incapacidades e objetivos de desenvolvimento" (GASPAR; FERREIRA; QUINTAS, 2017, p. 75). Assim sendo, a equoterapia é um tratamento alternativo viável (HENRIQUES, 2014), como também asseguraram os familiares cuidadores deste estudo e como foi ressaltado na pesquisa de
Barbosa e Van Munster (2013), os quais evidenciaram a influência da prática completar citada no desenvolvimento psicomotor de pessoas com necessidades especiais.

Montar o cavalo, possibilita ao indivíduo desenvolver com o animal um relacionamento afetivo, que ativa o sistema límbico responsável pelas emoções, proporcionando motivação para se mover e repetir movimentos que seriam entediantes se aplicados de outra maneira (RODRIGUES; OLIVEIRA; ANDRI, 2015).

$\mathrm{Na}$ avaliação dos itens que julgam mais importantes para o futuro do filho (expectativas), pôde-se perceber que os cuidadores afirmaram a necessidade de reabilitação, bem-estar, sucesso na escola, autonomia na vida diária, felicidade, normalidade, cuidados pessoais e amigos (interação social).

Em relação expectativas sobre o futuro, estudos indicam que há intercessão de demandas comuns às famílias, independente de qual seja a deficiência da criança, como a necessidade de orientações, informações e serviços, e outras que normalmente são características e associadas aos obstáculos que as crianças enfrentarão e à etapa do desenvolvimento infantil em que se encontram (NEVES; SILVEIRA, 2013; MARTINS; MONTEIRO; GONÇALVES, 2016).

Quanto às expectativas relacionadas às redes de apoio mais relevantes para o futuro do filho, os familiares cuidadores julgaram ser de muita importância à família, os profissionais e eles próprios.

Barbosa et al. (2016) relatam que as famílias e as crianças com necessidades especiais precisam de uma rede social e de apoio bem estruturada para que possam seguir o curso natural da vida com qualidade. A rede social refere-se ao âmbito estrutural ou institucional, ligada a uma pessoa. É uma estrutura em que as relações conectam os vários indivíduos proporcionando que os recursos de apoio fluam a partir de vínculos. Quanto à rede de apoio, é um sistema em que há interação entre pessoas ou grupos e a partir de contatos entre eles constroem vínculos de amizade e de informação, recebendo apoio material, emocional, afetivo, colaborando para o bem-estar mútuo e edificando fatores positivos na prevenção de agravos e manutenção da saúde.

Por conseguinte, quanto à avaliação das emoções sentidas (sentimentos) com frequência, relacionadas ao futuro do filho, familiares cuidadores relataram apresentar uma série de sentimentos como ansiedade, sentido de proteção, infelicidade, tristeza, insegurança, entre outros. Contudo, os mais presentes foram ansiedade e o sentimento de proteção.

Investigações asseveram que diante do processo de adoecer de um filho, particularmente com a caracterização da necessidade especial, os familiares cuidadores são tomados por sentimentos de ansiedade, angústia e impotência (FIRMINO; SOUSA, 2013; LAU et al., 2014). Outra questão ponderada foi o instinto de proteção materno, o qual foi reforçado em outra pesquisa (MARQUES; CAVALCANTI; RUZZI-PEREIRA, 2015). 
Destarte, conhecer os sentimentos experimentados pelos cuidadores é de grande importância já que eles podem exercer influência positiva ou não, que poderá potencializar ou dificultar o processo de melhoria da qualidade de vida da criança (LIMA; FENSTERSEIFER, 2017).

\section{CONCLUSÃO}

Conforme os achados, conviver com crianças portadoras de necessidades especiais gera nos cuidadores uma série de sentimentos e expectativas causadas por dilemas e dúvidas em relação à vida e o futuro da criança. Sobre os sentimentos, destaque para a ansiedade, sentido de proteção e tristeza. Quanto às expectativas, enfatizaram sobremaneira o desejo de que haja a reabilitação, bem-estar, sucesso na escola, autonomia e felicidade.

\section{REFERÊNCIAS}

ANDRADE, G. P. da S.; CUNHA, M. M. A importância da equoterapia como instrumento de apoio no processo de ensino e aprendizagem de crianças atendidas nesta modalidade terapêutica. REP's, Mato Grosso, v. 2, n. 5, p. 132-142, 2014.

BARBOSA, G. de O.; VAN MUNSTER, M. de A. Influência da equoterapia no desenvolvimento psicomotor de pessoas com necessidades especiais. Rev. Educ. Especial, Santa Maria, v. 46, n. 26, p. 451-464, 2013. DOI: http://dx.doi.org/10.5902/1984686x. Disponível em: <https:// periodicos.ufsm.br/educacaoespecial/article/view/4839/pdf $>$. Acesso em: 30 fev. 2018.

BARBOSA, T. A. et al. Support network and social support for children with special health care need. Rev. RENE, Fortaleza, v. 17, n. 1, p. 60-66, 2016. DOI: http://dx.doi.org/10.15253/2175-6783.2016000100009.

CHOON, M. A. E. S. G. Os benefícios da Equitação Terapêutica no desenvolvimento cognitivo e motor de crianças com Necessidades Educativas Especiais. 2015. 155 f. Tese (Mestrado) - Curso de Atividade Física Adaptada, Faculdade de Desporto Universidade do Porto, Porto, 2015.

DIGUISEPPI, C. G. et al. Demographic profile of families and children in the Study to Explore Early Development (SEED): Case-control study of autism spectrum disorder. Disabil Health J., [s.I], v. 9, n. 3, p. 544-551, 2016. DOI: http://dx.doi.org/10.1016/j.dhjo.2016.01.005>.

FEDERAÇÃO NACIONAL DE COOPERATIVA DE SOLIDARIEDADE SOCIAL (FENACERCl, 2006). Questionário para a família. Disponível em: <http:// www.aiasbo.it/download/daphne/Quest_Parents_Portugal.pdf>. Acesso em: 15 out. 2017.

FIGUEIREDO, S. V.; SOUSA, A. C. C. de; GOMES, I. L. V. Menores com necessidades especiais de saúde e familiares: implicações para a Enfermagem. Rev. Bras. Enferm., Brasília, v. 69, n. 1, p. 88-95, 2016. FapUNIFESP (SCiELO). DOI: http://dx.doi.org/10.1590/0034-7167.2016690112i.

FIRMINO, C. D. B.; SOUSA, M. N. A. Sentimentos e vivências de familiares em frente ao diagnóstico de câncer na criança. Rev. Bras. Pesq. Saúde, Vitória, v.15, n. 2, p. 6-12, 2013.

GASPAR, C.; FERREIRA, D.; QUINTAS, S. Terapeutas de 4 patas-terapia assistida por animais em unidades de multideficiência. Rev. Est. Inv. Psico. Educ., Espana, n. 11, p. 075-079, 2017.

GÓES, F. G. B.; CABRAL, I. E. Discursos sobre cuidados na alta de crianças com necessidades especiais de saúde. Rev. Bras. Enferm., Brasília, v. 70, n. 1, p. 163-171, 2017. DOI: http://dx.doi.org/10.1590/0034-71672016-0248.

HENRIQUES, M. J. dos S. R. Os benefícios da equitação terapêutica no desenvolvimento cognitivo e motor de crianças com necessidades educativas especiais. 2014. 113 f. Tese (Doutorado) - Curso de Educação Especial: Domínio Cognitivo-motor, Escola Superior de Educação João de Deus, Lisboa, 2014.

LAU, S. et al. Family life events in the first year of acute lymphoblastic leukemia therapy: A children's oncology group report. Pediatr. Blood Cancer, Hoboken, v. 61, n. 12, p. 2277-2284, 2014. DOI: http://dx.doi. org/10.1002/pbc.25195.

LIMA, R. C. de; FENSTERSEIFER, L. Psicodiagnóstico infanto-juvenil: como as expectativas dos cuidadores influenciam a vivência do processo de avaliação. Rev. Gra. Psicol. Puc Minas, Minas Gerais, v. 2, n. 3, p. 113128, 2017. Disponível em: <http://periodicos.pucminas.br/index.php/ pretextos/article/view/14251/11201>. Acesso em: 25 mai. 2018.

MANOEL, M. F. et al. As relações familiares e o nível de sobrecarga do cuidador famliar. Esc. Anna Nery Rev. Enferm., Rio de Janeiro, v. 17, n. 2, p. 346-353, 2013.

MARQUES, L. N.; CAVALCANTI, A.; RUZZI-PEREIRA, A. O viver com a doença falciforme: percepção de adolescentes. Rev. Ter. Ocup., São Paulo, v. 26, n. 1, p. 109-117, 2015. DOI: http://dx.doi.org/10.11606/ issn.2238-6149.v26i1p109-117.

MARTINS, M. M.; MONTEIRO, M. C. D.; GONÇALVES, L. H. T. Vivências de familiares cuidadores em internamento hospitalar: o início da dependência do idoso. Rev. enferm. UFPE on line, Recife, v. 10, n. 3, p. 1109-1118, 2016.

MENDES, M. E. S. T. Educação inclusiva e multideficiência: a pessoa, o sonho, a realidade. Inc. Soc., Brasília, v. 11 n. 1, p. 129-137, 2017.

NEVES, E. T.; SILVEIRA, A. da. Challenges for family caregivers of children with special health care needs: contributions of nursing. JNUOL, Recife, v. 7, n. 5, p. 1458-1462, 2013. ISSN 1981-8963. Disponível em: <https://periodicos.ufpe.br/revistas/revistaenfermagem/article/ view/11633/13704>. Acesso em: 23 mai. 2018. DOI: https://doi. org/10.5205/1981-8963-v7i5a11633p1458-1462-2013.

NIJS, S.; VLASKAMP, C.; MAES, B. The nature of peer-directed behaviours in children with profound intellectual and multiple disabilities and its relationship with social scaffolding behaviours of the direct support worker. Child Care Health Dev., Oxford, v. 42, n. 1, p. 98-108, 2016. DOI: http://dx.doi.org/10.1111/cch.12295.

NOHARA, S. S. B. et al. Atuação fisioterapêutica na sobrecarga física e dor de cuidadores de crianças com paralisia cerebral. RBPS, Fortaleza, v. 30, n. 4, p. 1-7, 2017. DOI: http://dx.doi.org/10.5020/18061230.2017.6461.

OLIVEIRA, A. K. C. de; MATSUKURA, T. S. Estresse e apoio social em cuidadores de crianças com paralisia cerebral. Cad. Ter. Ocup. UFSCar, São Carlos, v. 21, n. 3, p. 493-503, 2013. DOI: http://dx.doi.org/10.4322/ cto.2013.051.

PAVÃO, S. L. Efeito da orientação domiciliar no desempenho funcional de crianças com necessidades especiais. Redalyc, México, v. 7, n. 1, p. 21-29, 2011. DOI: http://dx.doi.org/10.1590/0034-7167-2016-0248.

RODRIGUES, M. da S.; OLIVEIRA, E.; ANDRI, S. Equoterapia na reabilitação neuropsicológica do individuo com a síndrome do X-frágil. 2015. 10 f. Dissertação (Mestrado) - Curso de Psicologia, Universidade do Contestado, Monte Carlo, 2015. Saúde, Vitória, v. 15, n. 2, p. 6-12, 2013.

SILVA, O. M. P. da; PANHOCA, L.; BLACHMAN, I. T. Os pacientes portadores de necessidades especiais: revisando os conceitos de incapacidade, deficiência e desvantagem. Salusvita, Bauru, v. 23, n. 1, p. 109-116, 2004.

SUSKIND, D. L. et al. Project ASPIRE. Otol. Neurotol., Hagerstown, v. 37 , n. 2, p. $110-117,2016$. DOI: http://dx.doi.org/10.1097/ mao.0000000000000931. 
TOIGOA, T.; JÕNIORB, E. C. P. L.; ÁVILA, S. N. O uso da equoterapia como recurso terapêutico para melhora do equilíbrio estático em indivíduos da terceira idade. Rev. Bras. Geriatr. Gerontol., Rio de Janeiro, v. 3, n. 11, p. 391-403, 2008.
ZAMO, C. da G. de S.; ZAMO, R. de S. Bioética, ética e equoterapia. Rev. Bras. Equoterapia, Porto Alegre, v. 1, p.1-5, 2015. Disponível em: <http://http://equoterapia.org.br/media/artigos-academicos/documentos/28042053.pdf>. Acesso em: 25 out. 2017.

Submetido em: $14 / 06 / 2018$

Aceito em: 06/09/2019 